\title{
El valor de uso y la crítica total de la sociedad moderna en la lectura de El capital, de Marx, que propone Bolivar Echeverría
}

\author{
David Moreno Soto
}

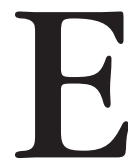

1 ensayo "La 'forma natural' de la reproducción social", ${ }^{1}$ publicado en 1984 -y luego en una nueva versión, con el título "El valor de uso: ontología y semiótica",$-^{2}$ tiene una importancia especial en la obra de Bolívar Echeverría. Se trata de un texto programático, en el que su autor formula con precisión el eje en torno al cual han girado sus investigaciones hasta ese momento y traza el rumbo que posteriormente han de seguir.

Se trata, dice Echeverría de entrada en aquella primera versión de su ensayo, de una contribución a "la reconstrucción del concepto crítico radical de valor de uso" que permitiría "mostrar la falta de fundamento de aquella identificación del marxismo con el productivismo occidental, el progresismo economicista del capitalismo y el estatalismo político burgués que llevó a plantear [...] la inadecuación del discurso marxista con las exigencias de la nueva figura histórica de la revolución".

Así establece Echeverría el terreno de la discusión en la que está interviniendo. Define su objetivo como el de refutar la falsa identificación del pensamiento de Marx con ideas que tanto marxistas como pensadores e ideólogos no marxistas se han hecho de ese pensamiento en su intento de interpretarlo, sea para retomarlo o para criticarlo. Esta reconstrucción, insiste Echeverría, vendría a poner en claro "lo que en verdad tiene Marx [...] que decir en la discusión actual de los fundamentos de la actividad política revolucionaria”.

Bolívar Echeverría precisa que su interlocutor principal en esta discusión es Karl Korsch, quien, hacia 1950, hizo originalmente este planeamiento crítico, que luego fue plagiado y reformulado de modo vulgar en los años setentas.

${ }^{1}$ B. Echeverría, Cuadernos Políticos. México, Era, núm. 41, julio-diciembre, pp. 33-46.

${ }^{2}$ B. Echeverría, Valor de uso y utopía. México, Siglo XXI, 1998. 
La interlocución con Karl Korsch seguirá desempeñando un papel central en la evolución del pensamiento de Echeverría, en su propuesta de lectura de la crítica de la economía política y, en general, en la perspectiva desde la que entiende lo que es el marxismo y su lugar en la historia moderna.

Baste recordar aquí que Korsch -a quien Bertolt Brecht consideraba su maestro de marxismo, y que influyera también profundamente a Walter Benjamin, ambos tan caros a Echeverría- es autor de Marxismo y filosofía, uno de los dos libros fundacionales del marxismo crítico, junto con Historia $y$ conciencia de clase, de Georg Lukács, ambos publicados en 1923. Estos libros establecieron las bases y el marco dentro del cual surgieron los aportes centrales del pensamiento crítico del siglo xx como los de la Escuela de Fráncfort.

Kart Korsch fue quien intentó pensar con la mayor radicalidad las experiencias revolucionarias más profundas del siglo XX -la de Alemania en 1918-1923 y la rusa que culmina en 1917- y la relación de estas experiencias con la obra de Marx y con las interpretaciones que los marxistas hacían de ella. En esta vía, Korsch retoma la tradición crítica inaugurada por Rosa Luxemburgo y continuada por el movimiento consejista europeo contra las versiones "oficiales" del marxismo, tanto del revisionismo reformista socialdemócrata como la del bolchevismo revolucionario leninista, y en esta reflexión llega a concebir la necesidad de emprender una relectura sistemática del conjunto de la obra de Marx y Engels, la más ambiciosa que se haya intentado hasta hoy, en su obra Karl Marx -publicada primero en 1938 y en múltiples ocasiones retrabajada por su autor posteriormente.

Podemos reconocer huellas profundas de la reflexión korschena tanto en los aportes clave de Echeverría al desarrollo de la crítica de la economía política como en su visión de la relación del marxismo con las experiencias revolucionarias del siglo $\mathrm{xx}$, así como en el refinado rigor con el que construye una ortodoxia crítica orientada a disolver todo dogmatismo en la interpretación de los conceptos fundamentales de la crítica de la economía política.

Desde el análisis de la contradicción del valor y el valor de uso, y su desarrollo como relación entre estructura y configuración del proceso de trabajo, y entre forma natural y forma de valor del proceso de reproducción social, hasta su teoría de lo político como función sintética de la socialidad y su teoría del cuádruple ethos, las investigaciones de Echeverría se desarrollan en plena correspondencia con la tesis korscheana de que "el primer principio básico de la nueva ciencia revolucionaria de la sociedad es el principio de la especificación histórica de todas las relaciones y circunstancias sociales". 3

Éste es, pues, el horizonte teórico-político en el que Bolívar Echeverría define en ese entonces el eje de toda su actividad como investigador, autor

\footnotetext{
${ }^{3}$ Karl Korsch, Karl Marx. Barcelona, Ariel, 1975, p. 25.
} 
y profesor, sus proyectos editoriales, sus trabajos de traducción, sus participaciones en conferencias, coloquios y seminarios, sus cursos académicos, etcétera. Así entendía en 1984 su labor como el medio a través del cual sería posible abrir "el discurso de Marx ante los nuevos problemas de la revolución contemporánea”. Para él ésta era la tarea teórica más importante de los marxistas en la época actual.

En su ensayo "La forma natural de la reproducción social", se propone poner al día los conceptos fundamentales del marxismo en confrontación con los desarrollos contemporáneos de las ciencias sociales en una línea de ortodoxia rigurosa y expone su descubrimiento de la dimensión semiótica de la reproducción social que será la base de sus aportes a la teorización crítica de la cultura y la política. En las siguientes dos décadas tematiza estos aportes en ensayos de interpretación del capitalismo contemporáneo que hacen época en el pensamiento crítico contemporáneo.

Como no voy a referirme al conjunto del ensayo, remito al comentario que le hizo Jorge Veraza publicado en 1985. ${ }^{4}$ Veraza reconoce -y de hecho retomará críticamente en su propia obra- la propuesta programática de Echeverría para el desarrollo del pensamiento crítico contemporáneo y reseña puntualmente los referentes polémicos explícitos e implícitos que enmarcan su texto, así como los problemas que se ponen en juego en esa discusión. En su comentario, que es la primera parte de un trabajo más extenso aún inédito, Veraza adelanta su crítica al ensayo que, por cierto, es muy similar a la que el mismo Echeverría hace a Marx, es decir, que no presenta el contenido del valor de uso, pues su perspectiva de análisis permanece formal. ${ }^{5}$

Aquí sólo quiero llamar la atención sobre un aspecto particular del ensayo, el relativo al cambio de su propia perspectiva respecto al sentido de su intervención. Insisto en que este empeño en "la reconstrucción del contenido del concepto" de valor de uso o forma natural "y de su efectividad crítica para el presente" fue una constante a lo largo de la vida de Bolívar Echeverría en México, por lo que este ensayo -en sus dos versiones, y tomando en cuenta el cambio de perspectiva al que me refiero- debe considerarse también, digo, como una guía de lectura de su obra, tanto de la anterior como de la ulterior.

Esta reconstrucción es posible, dice Echeverría, porque aunque en la obra de Marx no encontramos tematizado ese contenido del valor de uso, sin embargo, "su lugar y su medida están allí: demarcados por la radicalidad de la

${ }^{4}$ Jorge Veraza, "A propósito de 'La «forma natural» de la reproducción social' de Bolívar Echeverría”, en Itaca, núm. 3, primavera, 1985, pp. 23-28.

5 Para Veraza, "Bolívar Echeverría retoma el programa korscheano" -es decir, "desarrollar la teoría revolucionaria del presente" - "pero critica su error" (confunde a Marx con el "marxismo") "y precisa el lugar de la indagación y desarrollo del marxismo: el tema del valor de uso" (ibid., p. 24). 
crítica de Marx al capitalismo". Y bien, ¿hasta dónde llega esta radicalidad?, ¿cuáles son sus alcances? Echeverría señala dos extremos.

La radicalidad del discurso crítico de Marx llega explícitamente -por un lado- "hasta el cuestionamiento de la forma en que tanto el sujeto como la objetividad se constituyen en la época moderna, y plantea, por tanto, una idea de revolución que, lejos de quedar atrapada en las ilusiones del siglo pasado, implica una propuesta cuya plena validez sólo ha podido mostrarse a la luz de las desilusiones del presente". Por otro lado, este concepto crítico de "forma natural" o valor de uso, que "hace estallar el horizonte de inteligibilidad en el que se mueve el pensamiento moderno", "ocupa un lugar central en el discurso de Marx" porque es el concepto que le permite a este discurso "precisar el sentido de su trabajo crítico", de modo que sin él "resultan impensables [...] tanto la crítica particular del comportamiento y el discurso económicos de la época capitalista como aquella otra, general, la crítica de la totalidad de la vida social moderna".

Bolívar Echeverría llama de forma natural "concepto de contraste", porque es el que permite caracterizar suficientemente la peculiaridad del modo capitalista de producción y del modo de vida que se estructura en torno de él, su carácter histórico, el hecho de que se trata de un modo de vida posible entre otros, que hubo un antes y un después de él en donde no existía ni por tanto podrá seguir existiendo; que no es, pues, natural sino histórico, perecedero. Pero también es, por lo tanto, el concepto que describe la estructura básica de la vida social humana, es decir, aquello que la revolución debe liberar, el contenido reprimido, distorsionado, explotado por la forma capitalista de la vida social y sobre el cual ésta se monta parasitariamente. Es, en fin, el concepto que refiere el objeto y, por tanto, la meta de la revolución proletaria comunista.

Este alcance crítico radical del concepto de valor de uso en el discurso crítico de Marx es subrayado implícitamente en el epígrafe -también de las "Glosas a Wagner" - con el que encabeza el primer avance publicado de su lectura de la obra de Marx, "Comentario sobre el 'punto de partida' de El capital", publicado originalmente en 1977.6

${ }^{6}$ Véase B. Echeverría, "Comentario sobre el 'punto de partida' de $E l$ capital", en $E l$ discurso crítico de Marx. México, Itaca/FCE, 2015, pp. 93-121. El epígrafe es el siguiente: "Sólo un vir obscurus que no ha entendido ni una palabra de $E l$ capital puede concluir que, en [mi] obra, el valor de uso no desempeña ningún papel [...]. En mi teoría, el valor de uso desempeña un papel importante de un orden totalmente diferente al que ha tenido en la economía anterior" (K. Marx, "Notas a Wagner", en MEW, t. 19, pp. 369 y 371). 
En su comentario, Echeverría invita a considerar "el conjunto de la argumentación desarrollada" en los cuatro primeros capítulos de El capital. Se trata, dice, de "una recomendación implícita del autor mediante el orden que sigue en su exposición”. Así, a través de la reconstrucción sistemática de ese orden expositivo, concluye que "la contradicción inherente a la forma mercantil de los objetos prácticos [...] actúa en la cotidianidad de los individuos sociales privados; los acosa imperceptiblemente: les hace la vida imposible".

En otros términos, Echeverría presenta "la manera" en que Marx analiza la estructura de la forma mercancía como el punto de partida de una crítica económica de la sociedad moderna, pero que llega hasta la cotidianidad de la misma, es decir, precisamente, hasta "la crítica de la totalidad de la vida social moderna"; e indica, en el epígrafe que encabeza su comentario, que este alcance de la crítica de la economía política se revela a la luz del "importante papel" que desempeña el valor de uso en la teoría de Marx. Ésta es de hecho la primera referencia al programa de trabajo centrado en la reconstrucción del concepto crítico radical de valor de uso que ocho años después, ya concluida la etapa más intensa de su lectura de El capital, plantea de modo explícito en el ensayo "La forma natural de la reproducción social".

Como vemos, ya en su "Comentario sobre el punto de partida de $E l$ capital”, está intentando ir más allá de los esfuerzos de los marxistas del siglo XX por trascender las lecturas economicistas de la obra de Marx y desarrollar la crítica económica del capitalismo hacia la crítica de la cultura, la política y la civilización burguesas, y para ello intenta mostrar cómo es que estas críticas efectivamente están allí, implícitas en el análisis crítico de las formas de movimiento de la riqueza burguesa que lleva a cabo Marx en el texto de El capital, y la clave que puede volverlas explícitas es precisamente el concepto crítico radical de valor de uso. Esta concepción acerca del alcance del concepto de valor de uso se irá precisando a través de una rigurosa lectura de El capital sistemática y largamente razonada en la que Bolívar Echeverría desentraña la profunda radicalidad de ese concepto que se despliega en el texto de Marx.

Pero es también una lectura que resume críticamente un cúmulo de investigaciones y debates acerca de los lineamientos y premisas teóricas, metodológicas, literarias, hermenéuticas, etcétera, resultado de los esfuerzos de generaciones de investigadores y pensadores a lo largo del siglo xx en torno a la obra Marx y que supone un conocimiento reflexionado de la literatura europea en la que se sintetizan aquellos esfuerzos y que Echeverría conociera de primera mano al calor de intensos debates con los estudiantes alemanes movilizados. Él lee esta literatura y la discute junto con los textos fundacionales del marxismo crítico de Rosa Luxemburgo, Karl Korsch, Georg Lukács, etcétera, que habían sido enterrados por la ola reaccionaria de la Guerra fría, y junto con los acontecimientos históricos de la coyuntura como la Revolución 
cubana, la guerra de Vietnam, la gesta del Che Guevara y, en general, los movimientos de liberación nacional del Tercer Mundo. ${ }^{7}$ Para aquellos estudiantes radicales, la revolución comunista está a la orden del día y ellos creen vivirla en el centro de la misma.

El proyecto de lectura del texto de $E l$ capital que surge en este clima dominado por la creencia de que es inminente la revolución mundial llega a un momento culminante en el conjunto de los ensayos compilados en $\mathrm{El}$ discurso crítico de Marx, publicado en 1986, aunque la redacción de los ensayos data de 1974 a 1980 y el prólogo está fechado en 1984 -es decir, el mismo año de la publicación de "La forma natural de la reproducción social". Pero esta lectura también se puede reconocer, implícitamente, en su edición y traducción del manuscrito preparatorio de la crítica de la economía política de 1861-1863 sobre la "subsunción formal y subsunción real del proceso de trabajo al proceso de valorización”, publicado en $1983 .{ }^{8}$

También en 1983 Echeverría resume el eje de su lectura de la crítica de la economía política en una conferencia con motivo del centenario de la muerte de Marx en la que expone la contradicción del valor y el valor de uso como "la contradicción que sostiene a todas las contradicciones del mundo moderno". Allí argumenta que "la intención crítica de Marx con respecto a la sociedad moderna" se expresa en las distintas figuras de esta contradicción - "a la que le corresponde propiamente el término técnico de contradicción entre la forma natural y la forma de valor del objeto mercantil"- que se despliegan en los distintos niveles del argumento del texto de El capital. ${ }^{9}$

\section{III}

Lo dicho hasta aquí confirma la afirmación inicial de que el ensayo "La forma natural de la reproducción social" es una formulación programática madura

${ }^{7}$ Sobre esta síntesis y el clima en el que tiene lugar, véase Andrés Barreda, "Aproximación a la crítica de la economía política de Bolívar Echeverría", en Raquel Serur, comp., Bolívar Echeverría. Modernidad y resistencias. México, UAM-Xochimilco/Era, 2015, así como B. Echeverría, "Sobre la muerte del Che Guevara", en Calibán. Revista Semestral de Antropología Cultural [Trad. y presentación de Javier Sigüenza].

${ }^{8} \mathrm{~K}$. Marx, "Subsunción formal y subsunción real del proceso de trabajo al proceso de valorización (inédito)", en Cuadernos Políticos, núm. 37, julio-septiembre, 1983, pp. 2-14. Publicado luego como K. Marx, La tecnología del capital. Subsunción formal y subsunción real del proceso de trabajo al proceso de valorización (extractos del Manuscrito 1861-1863). México, Itaca, 2011.

${ }^{9}$ B. Echeverría, La contradicción del valor y el valor de uso en $\mathrm{El}$ capital, de Karl Marx. México, Itaca, 1994. 
del proyecto de trabajo en el que se ocupara Echeverría durante las cuatro décadas que viviera en México.

Encontramos una primera enunciación del sentido global de este proyecto en una nota a pie de página del trabajo que presentó en 1974 como tesis de licenciatura con el título "Apuntes para un comentario de las "Tesis sobre Feuerbach'”. En esa nota se indica que este texto es "parte de otro más amplio en el que intentamos, dice Echeverría, estudiar de manera especial la relación entre la revolución teórica de Marx y el proceso de constitución del movimiento proletario en movimiento comunista". Más aún, al año siguiente, en 1975, se publica este ensayo con el título "La revolución teórica comunista en las Tesis sobre Feuerbach". ${ }^{10}$

Así pues, este proyecto de reconstrucción de la teoría de la revolución comunista en el que Echeverría entiende que se enmarca su trabajo teórico diez años después, en la versión de su ensayo de 1984, se traduce en la reconstrucción de la centralidad del valor de uso en el discurso crítico. Así, para Echeverría, la reconstrucción de este concepto crítico radical de valor de uso presenta dos vertientes, o más bien una misma que corre por dos vías: la de la crítica de la forma moderna capitalista de la vida social y la de la construcción de la plataforma teórica de la estrategia de la revolución proletario-comunista de esa forma de vida. En otros términos, este concepto de valor de uso vincula la crítica de la economía política y el materialismo histórico, y por eso es la clave de la crítica global de la civilización burguesa, el concepto que le da unidad al discurso crítico de Marx y lo constituye como una teoría de la revolución comunista, del que depende su "efectividad crítica para el presente".

Bolivar Echeverría alude implícitamente a esta conexión entre crítica económica y crítica total de la sociedad moderna cuando refiere en su ensayo a los Manuscritos de París, de 1844. En estos manuscritos, en efecto, se plantea explícitamente -en el prólogo- la crítica de la economía política como parte del proyecto teórico global de crítica de todas las esferas de la vida social burguesa: "del derecho, de la moral, de la política, etcétera", y, sobre todo, en el pasaje titulado "El trabajo enajenado", así como el programa para desarrollar esta crítica económica que se desprende de esta conexión: el desarrollo del sistema teórico de la ciencia económico como el sistema de la propiedad privada o sistema de la enajenación total. ${ }^{11}$

${ }^{10}$ En El materialismo de Marx (México, Itaca, 2000) se recogen las variantes de las distintas versiones de este trabajo.

11 "La economía política parte del trabajo como la verdadera alma de la producción, y, sin embargo, no da nada al trabajo, y a la propiedad privada se lo da todo [...] nosotros vemos que esta aparente contradicción es en realidad la contradicción del trabajo enajenado consigo mismo y que la economía política se limita a proclamar las leyes del trabajo enajenado [...] podemos desarrollar todas las categorías de la economía política y 
Echeverría es consciente de esta relación, pues su propuesta de lectura del texto de El capital fue preparada, además de por su comentario a las Tesis sobre Feuerbach, por su esmerada traducción y edición de los Cuadernos de París -contemporáneos de los Manuscritos de 1844-publicada en $1974 .{ }^{12}$

Cabe insistir en que, al preparar su lectura del texto de El capital mediante la recuperación del joven Marx de las Tesis sobre Feuerbach y los Cuadernos y los manuscritos de París, es consciente de que está contribuyendo a los esfuerzos de generaciones de pensadores y luchadores por reflexionar desde el discurso crítico de Marx las experiencias revolucionarias del siglo xx, su empeño por trascender las lecturas economicistas de ese discurso y captar los elementos conceptuales que brinda la teoría de Marx en su unidad, desde los escritos de juventud hasta $E l$ capital, para pensar críticamente, desde sus fundamentos, la civilización capitalista.

\section{IV}

En lo que sigue quisiera dejar planteado un problema implícito en la discusión a la que nos convoca Bolívar Echeverría cuando reescribe su ensayo, una década después, introduciendo en él algunas modificaciones, en verdad pocas y de matiz, pero profundamente significativas, y lo publica en 1998, ahora con el título "El valor de uso: ontología y semiótica", en el libro Valor de uso y utopía.

En la presentación al volumen, señala la importancia de esta nueva versión de su ensayo al indicar que se encuentra en la base del conjunto de ensayos compilados en el libro y que de hecho fue redactado con anterioridad a aquéllos; no precisa cuándo lo escribió, pero debió ser cuando menos antes de 1994, que es la fecha de los más antiguos de ellos.

Así pues, tras diez años de reflexión, continúa reelaborando el eje central de su pensamiento. Sin embargo, en la nueva versión de su ensayo sobre el valor de uso encontramos, como decía, una serie de modificaciones significativas que, como el conjunto de los ensayos compilados en el volumen, tienen la intención de provocar un debate, pues plantean un problema que pone en cuestión aspectos centrales de su posición teórica.

Resumamos la idea central: en primer lugar, encontramos que cambia el terreno de la discusión en la que interviene con la nueva versión de su ensayo

en cada una de ellas [...] nos encontraremos de nuevo con una determinada expresión, ya desarrollada, de estos primeros fundamentos": "el trabajo enajenado" y "la propiedad privada" (C. Marx, "Manuscritos económico-filosóficos de 1844", en Marx. Escritos de juventud. México, FCE, 1987, pp. 603-604).

${ }^{12}$ K. Marx, Cuadernos de París. Notas de lectura de 1844. México, Era, 1974 [México, Itaca, 2009]. 
sobre el valor de uso. A hora ya no se trata de lo que "tiene Marx [...] que decir en la discusión actual de los fundamentos de la actividad política revolucionaria", sino "de los fundamentos de una nueva práctica de la política"; o, también, la cuestión ya no es, dice, abrir "el discurso de Marx ante los nuevos problemas de la revolución contemporánea", sino "ante los nuevos problemas de la política contemporánea”. Así pues, la revolución le deja su lugar a la política.

Más aún, se ha perdido de vista la interlocución fundamental con Karl Korsch y la preocupación por "mostrar la falta de fundamento de aquella identificación del marxismo con el productivismo occidental, el progresismo economicista del capitalismo y el estatalismo político burgués que llevó a éste a plantear [...] la inadecuación del discurso marxista con las exigencias de la nueva figura histórica de la revolución”. En lugar de esta discusión con Korsch, parece pasar a primer plano la interlocución, que en la versión de 1984 era secundaria, con Michel Foucault y con Jean Baudrillard. ${ }^{13}$

Pero, además de este desplazamiento del terreno de la discusión y, por tanto, de los interlocutores de Bolívar Echeverría, encontramos un cambio aún más drástico de su posición en uno de los ensayos compilados en el mismo volumen bajo el título de "Modernidad y revolución", escrito en 1997, después de la nueva versión del ensayo sobre el valor de uso, y sobre la base de éste, como sugiere en la presentación del libro. En este texto vemos que Echeverría suscribe, pero ahora de la mano de la Escuela de Fráncfort, y reformulada como "un Marx acrítico ante la idolatría de la técnica" (p. 65), aquella misma identificación del pensamiento de Marx "con el productivismo [...], el progresismo economicista [...] y el estatalismo [...] burgués”, que diez años antes quería refutarle a Karl Korsch.

Una identidad que, hay que decirlo, aunque sea de paso, a principios del siglo XXI no deja de seguir siendo vulgarizada, sino, al contrario, ahora con nuevos bríos, como una vulgarización de la vulgarización de los criterios que sin embargo debieran orientar los esfuerzos, por demás urgentes, de entender racionalmente, es decir, en su unidad, los múltiples aspectos de la crisis actual en la que se acumulan y se retroalimentan los desastres ambientales junto con los económicos, culturales, políticos, etcétera.

13 "La incomprensión de lo alcanzado por Marx, que empobrece la magnífica obra de Foucault, puede justificarse por la escasa cercanía de este autor al texto de El capital. No sucede lo mismo con la incomprensión voluntaria de la que hace gala Jean Baudrillard, uno de los más agudos teóricos actuales del intercambio, la producción y el consumo" quien "le adjudica" a Marx "el más plano de los utilitarismos y descalifica todo lo que, en la línea de Marx pero más allá de él, pueda decirse acerca de un valor de uso dirigido a un disfrute más allá de los límites del do ut des" (B. Echeverría, "El valor de uso: ontología y semiótica", en Valor de uso y utopía. México, Siglo XXI, 1998, pp. 153-154, n. 1). En la primera versión, de 1984, dice: "[...] acerca de un valor de uso cuya aprehensión teórica trasciende necesariamente la metafísica de Occidente"). 
En realidad, Echeverría no abandona la interlocución fundamental con Karl Korsch, pues sus "Diez tesis sobre el marxismo", de 1950, en verdad continúan las ideas que plantea en "La crisis del marxismo", de 1931, y que a su vez retoman los miembros de la Escuela de Fráncfort; replantea el estatuto o el nivel en el que concibe la discusión con Korsch sobre el marxismo y cambia su posición en ella, pues ahora le da la razón a Korsch en su crítica a Marx, pero alineado del lado de la Escuela de Fráncfort y dándole a ésta todo el peso como interlocutora en la discusión que sin embargo, a través de ella, sigue entablando con Karl Korsch.

Así pues, ¿podríamos decir que nos encontramos ante un viraje en el pensamiento de Bolívar Echeverría?, o ¿se trata más bien del desarrollo consecuente de premisas que se hallan presentes ya en el texto de 1984?, o bien, incluso, ¿de ambas cosas a la vez?

Creo que lo que he dicho hasta aquí pone de manifiesto la importancia fundamental del diálogo crítico con Bolívar Echeverría que nos permita recuperar sus aportes al pensamiento crítico contemporáneo. Para contribuir a este diálogo crítico tan sólo quisiera señalar aquí dos problemas que me parecen deben ser motivo de ulteriores investigaciones.

En primer lugar, la perspectiva de la centralidad del concepto de valor de uso en el discurso crítico de Marx obliga a destacar la importancia de la definición de Marx en El capital cuando señala que "los valores de uso constituyen el contenido material de la riqueza, sea cual fuere la forma social de ésta”. Pues si por riqueza vamos a entender la constitución de la objetividad en la época moderna, ¿desde dónde va a llevarse a cabo el análisis crítico de esta objetividad si no es desde su contenido material?, es decir, desde esa dimensión básica sobre la cual se monta la forma o configuración que adopta históricamente para quedar subordinada al servicio del valor que se autovaloriza.

Toda la vida social moderna, centrada en torno a la explotación del plusvalor y subordinada a la acumulación de capital, se basa en la represión práctica y teórica, discursiva o significativa justamente de ese contenido y del sentido que le es inherente. Es la captación de ese contenido reprimido lo que permite percibir que son posibles otras formas de la condición humana y precisamente más humanas; más aún, cuál es el sentido y la medida de esa mayor o menor adecuación a lo humano, así como, por tanto, el sentido y medida de la inhumanidad de la forma de vida social existente.

Pero también se revela bajo nueva luz la presencia del valor de uso en la perspectiva analítica que se despliega a lo largo del texto de El capital, es 
decir, que en su conjunto lo que se expone allí es el sometimiento del sentido concreto de la existencia humana y la conversión de ésta en medio para la producción de plusvalor y la acumulación de capital, y que, por tanto, el desarrollo o perfeccionamiento en términos capitalistas de la civilización moderna sólo puede consistir en el perfeccionamiento de la refuncionalización del conjunto de las condiciones de la vida social como instrumentos o condiciones para la explotación de la clase obrera. Así lo revelan ya de entrada los dos epígrafes que encabezan el texto de Echeverría, que son citas del Capítulo vi inédito de $E l$ capital, y la cita, en nota a pie de página, de las "Glosas marginales al Tratado de economía política de Adolf Wagner". ${ }^{14}$

Estas referencias explican cómo es que, en efecto, el valor de uso, como "contenido material de la riqueza", es el concepto que articula toda la argumentación en los tres tomos de El capital, que lo que se expone allí es justamente el valor de uso del capital, el valor de uso que éste se ha apropiado y que por lo tanto se encuentra adaptado, subsumido, enajenado o refuncionalizado por el movimiento del valor que se valoriza. Todo el proceso material de producción, reproducción y desarrollo del capital no es otra cosa que el valor de uso funcionando como proceso de explotación y acumulación de plusvalor, es decir, el valor de uso subordinado como la base material de la producción, la economía y la civilización de la época moderna capitalista.

Sin embargo, esta dimensión de la crítica económica como crítica global de la sociedad burguesa que se desprende de las mencionadas referencias a dichos textos de Marx es limitada por la perspectiva de Echeverría, que solamente ve "cautela" en la indicación metodológica de Marx que restringe el tratamiento del valor de uso en su crítica de la economía política a la consideración del mismo "sólo allí donde juega un papel como categoría económica".

Para Bolívar Echeverría, esta restricción metodológica hace que en $E l$ capital el contenido del concepto de forma natural permanezca "en estado de incógnita" y que "el aporte central de Marx a una comprensión crítica de la modernidad" adolezca "de una disimetría o unilateralidad". Así justifica Echeverría la necesidad de su aporte a un esclarecimiento, sin el cual la crítica de la economía política, dice, "queda incompleta y en muchos sentidos enigmática”. Pienso que en esta apreciación crítica -que, sin embargo, es la que le permite introducir su concepción, también proveniente de Korsch, del

${ }^{14}$ Los epígrafes son las siguientes citas del Capítulo VI inédito de El capital: "Las únicas formas reales de las mercancías son sus figuras en el uso, sus formas naturales" y "[...] el proceso entero de trabajo en cuanto tal, en la interacción viva de sus elementos objetivos y subjetivos, se presenta como la figura total del valor de uso". No es ocioso señalar que en la segunda versión de su ensayo, Bolívar Echeverría suprime este segundo epígrafe. Por otro lado, Echeverría hace referencia a las "Glosas marginales al Tratado de economía política de Adolf Wagner" cuando alude a las "elucubraciones erráticas sobre las palabras 'valor' y 'valor de uso'". 
carácter negativo de la crítica de Marx-soslaya el problema principal: ¿qué significa que el valor de uso aparezca como categoría económica?

En otros términos, ¿un mero "concepto de contraste" pero "omnipresente en el texto de El capital" y, además, clave que "permite al discurso teórico precisar el sentido de su trabajo crítico"?

Creo que la desproporción entre la caracterización del concepto de valor de uso que propone Echeverría y la presencia y las funciones que le reconoce se desvanece si, leyendo sin prejuicios, observamos que la "omnipresencia" del concepto en el texto de Marx podría deberse a que, más que permitir precisar dicho "sentido crítico", lo indica positivamente.

Por otro lado, ya la mera insistencia reiterada con la que se repite la indicación metodológica de Marx en los Grundrisse (1857), en la Contribución a la crítica de la economía política (1859), en el Capítulo VI inédito (1859), en la primera edición de El capital (1867) y en las "Glosas a Wagner" (1882), de acuerdo con la cual el valor de uso "sólo allí donde juega un papel como categoría económica", ${ }^{15}$ indica que no se trata de un vacío provocado por un olvido o un descuido, como tienden a pensar otros lectores, ciertamente menos cuidadosos que Echeverría pero que creen seguirlo.

Pero tampoco se debe a una restricción impuesta por el carácter negativo de la crítica de Marx. Por el contrario, una lectura desprejuiciada debería tener en cuenta que a lo largo de veinticinco años de reflexión esta indicación no sólo se va haciendo más lacónica sino que acaba, en la edición final de El capital, en el mero señalamiento de los valores de uso como "contenido material de la riqueza, cualquiera que sea la forma social de ésta" y en la sociedad moderna "el soporte material del valor de cambio". Así pues, a Marx le parece cada vez menos necesario advertir a sus lectores cómo piensa tratar el valor de uso. Creo que esta intención, largamente meditada de Marx, de ahorrarle al lector explicaciones acerca de cómo piensa tratar el asunto y sustituirlas por el señalamiento directo de la cosa, difícilmente podría ser más elocuente.

¿No sería más prudente suponer, como hipótesis de investigación, que la indicación de Marx es rigurosamente adecuada a la perspectiva crítica desde la que describe el sistema económico en el que el "contenido material de la riqueza social" funciona bajo el dominio de la forma del valor como valor que se valoriza? Es decir, ¿que en esta forma de la riqueza, como en la sociedad que se estructura en torno de ella, el valor de uso no puede aparecer sino precisamente como la base oculta, reprimida, del movimiento autónomo del

15 "El valor de uso [...] sólo cae dentro del ámbito de consideración de la economía política cuando él mismo es determinación formal" (K. Marx, Contribución a la crítica de la economía política. México, Siglo XXI, 1980, p. 10). 
valor, base oculta pero fundante de la que sin embargo, y justamente por eso, se desprende la perspectiva crítica del análisis?

Si esta perspectiva puede ser crítica es porque ubica su punto de vista en ese contenido material para desde allí observar el objeto de su análisis, que es, precisamente, la forma social en que ese contenido se actualiza.

De acuerdo con nuestra hipótesis, ese contenido material enajenado no puede ser tematizado científicamente "en cuanto tal", "en positivo", en el análisis crítico de esta forma de la riqueza, sino justamente tal como aparece en el sistema económico que describe el movimiento de esta riqueza, es decir, como "categoría económica" o como elemento del sistema capitalista de relaciones económicas.

Por lo demás, Marx explica con más extensión la indicación metodológica crítica en cuestión en la página 540 de los Grundrisse a la que refiere en su ensayo y con más amplitud aún en el argumento que concluye con la idea del Capítulo VI inédito de El capital que Echeverría transcribe como epígrafe ("el proceso [...] de trabajo [...] en la interacción viva de sus elementos objetivos y subjetivos [...es] la figura total del valor de uso") en su primera versión de su ensayo "La forma natural de la reproducción social" -epígrafe que, como ya he señalado, se suprime en la nueva versión del ensayo. Creo que vale la pena reexaminar esos argumentos dentro de los cuales se encuentran las citas que nos refiere.

A la luz de estos señalamientos, podríamos reconocer el contenido del conjunto del plan de seis libros de la crítica de la economía política que Marx describe en la Contribución a la crítica de la economía política, de 1859, como el despliegue del concepto de valor de uso como la "base material en la cual se representa [...] el valor de cambio" y, por tanto, "el sistema de la economía burguesa" que constituye el objeto de estudio de toda la obra que Marx anuncia.

Un segundo problema que cabe señalar como otro tema pendiente de investigación es el siguiente: me parece reconocer una ambigüedad en la referencia de Bolívar Echeverría a los Manuscritos de París, en la que, como digo, alude a la conexión de la crítica económica con la crítica global de la civilización moderna. En su ensayo sobre la forma natural de la reproducción social (en las dos versiones) dice que en la obra de Marx "el contenido del concepto de forma natural permanece en estado de incógnita pese a los Manuscritos de 1844". O sea, que está sugiriendo que en estos Manuscritos no estaría ese Marx que retrata, desde la perspectiva de la Escuela de Fráncfort, en su texto de 1997 "Modernidad 
y revolución", es decir, ese Marx acrítico ante "la figura de lo que el ser humano ha hecho con el valor de uso [...] después de la revolución de las fuerzas productivas que abrió el camino a la modernidad", que es precisamente la figura "que sostiene al modo de producción capitalista"; ese Marx "fuertemente influido, en contra de su estirpe hegeliana, por la visión del progreso técnico propia del Iluminismo francés que permeaba al industrialismo inglés de su época"; un Marx que, por lo tanto, "no avanzaría en verdad en el camino de una crítica radical de la forma natural del mundo y de la vida en la época moderna".

Por lo tanto, el viraje no sería de Echeverría sino de Marx, quien estaría pasando de una fase propiamente crítica radical en su juventud a otra fase "positiva", o más bien positivista y, por lo tanto, acrítica o apologética del capitalismo, y esto debido, paradójicamente, a una recaída en la visión ultrarradical pero metafísica, mítica, "espontánea de revolución que es propia de la modernidad burguesa capitalista".

Esta "idea de revolución que emplea Marx" - afirma Echeverría-, como para rematar la paradoja, es "la misma que de alguna manera ha dominado en la historia del socialismo y del comunismo durante todo el siglo $\mathrm{xx}$, y que ha permeado en general todo el discurso político contemporáneo".

Así pues, en los años noventas, para Echeverría, comprender y aprovechar los verdaderos alcances del concepto de valor de uso hay que comenzar por reconstruir también la teoría de la revolución de la que originalmente forma parte ese concepto.

Hay que subrayar que tanto en su nueva versión del ensayo sobre el valor de uso como en "Modernidad y revolución", no renuncia a la idea ni a la práctica de la revolución. Más bien invita a la construcción de una nueva idea de revolución que sea adecuada a la nueva situación y, a la vez, al verdadero sentido y alcance del concepto crítico de valor de uso que está en la base del discurso crítico de Marx. Propone, pues, llenar un vacío en la obra de Marx, un vacío que Echeverría refiere como la tematización del valor de uso "en cuanto tal", "en positivo", y desde allí refundar el concepto de revolución. Por lo tanto, pareciera que quiere ir más allá de lo que él ve como conceptualización sólo negativa del valor de uso que Marx desarrolla en El capital.

Y aquí tenemos una vuelta más de la paradoja, pues al parecer a Bolívar Echeverría le estaría pareciendo insuficiente el discurso que es crítico por ser negativo, dependiente o parasitario del discurso burgués y que estuviera proponiendo otro tipo de discurso que, sin dejar de ser crítico, construyera científicamente el concepto positivo de forma natural que, dice, estaría ausente en la obra de Marx, pero, como digo, en la base de su pensamiento. ¿Es decir, que Echeverría podría estar hablando - tal vez sin sospecharlo- de un discurso crítico en la línea en que, por ejemplo, Adolfo Sánchez Vázquez desarrolla su concepción de la filosofía de la praxis? 
Por otro lado, quiere reconstruir el concepto crítico de valor de uso, pero ya no a partir de la idea de revolución que maneja Marx, que es la idea de revolución comunista que Echeverría suscribiera en sus primeros escritos publicados cuando menos hasta 1984, y que hasta aquel entonces se propusiera deslindar de la idea dominante y ya vulgarizada entre los marxistas, pero que diez años después le parece obsoleta.

Ahora, en 1994, propone llevar a cabo esa reconstrucción del concepto de valor de uso pero a partir de una nueva construcción de otra idea de revolución, diferente de la idea de Marx, que sí sería adecuada a la situación que se abre al final del siglo $\mathrm{xx}$, y construir esta nueva idea de revolución, no obstante, a partir del concepto marxiano de valor de uso, ese sí reconstruido.

En resumen, pues, insisto en que Echeverría no propone abandonar la idea de revolución sino refundarla. Plantea que el mismo concepto de valor de uso construido por Marx -en términos negativos o en tanto subordinado al capital- en el siglo XIX a partir da la idea comunista de revolución, ahora, en la vuelta del siglo XX al XXI, debe ser, a la inversa -y reconstruido en cuanto tal, en positivo-, la base para la construcción de una nueva idea de revolución. Por lo tanto, nos está invitando a repensar tanto la idea de revolución y la de valor de uso como la relación entre ambas y, por ende, el procedimiento para reconstruirlas, y, en fin, su propia idea de en qué consiste el carácter crítico del discurso marxista. Quizás por este camino reencontraríamos la vía hacia un posible libro intitulado no Valor de uso y utopía sino Valor de uso y revolución.

Valga, pues, esta provocativa invitación que nos dejara Bolívar Echeverría -en la conclusión de "Modernidad y revolución"- a acompañarlo en la búsqueda de "la idea de revolución que sería propia del tránsito civilizatorio en el que nos encontramos". 